\title{
Detection of metastatic fibro-lamellar type of hepato cellular carcinoma in ascitic fluid cytology in a liquid-based preparation
}

\begin{abstract}
Hepato cellular carcinoma (HCC) of fibro lamellar type is an uncommon primary hepatic carcinoma. They occur most commonly in young adults. Their etiology is not clear till date, as they are not associated with chronic liver disease. Fibro lamellar $\mathrm{HCC}$ is not an indolent tumor, but has a better prognosis than conventional HCC. We present an interesting case of metastatic fibro lamellar HCC detected in ascitic fluid prepared by liquid-based cytology (LBC). The diagnosis was confirmed by ultrasound-guided fine needle aspiration (US-FNA) of retroperitoneal lymph node. We report a rare case of fibro lamellar HCC detected on exfoliative cytology, which was confirmed by FNAC and immunochemistry (IHC).
\end{abstract}

Keywords: ascitic fluid cytology, fibro-lamellar hepato cellular carcinoma, fine needle aspiration, immunocytochemistry, liquid based cytology
Volume I Issue I - 2016

\author{
Nalini Gupta,' Anju Khariwa, ${ }^{2}$ Anupam Lal,' \\ Arvind Rajwanshi \\ 'Department of Cytology and Gynecological Pathology, \\ Postgraduate Institute of Medical Education and research, India \\ ${ }^{2}$ Department of Radio diagnosis, Postgraduate Institute of \\ Medical Education and Research, India
}

Correspondence: Nalini Gupta, Department of Cytology and Gynecological Pathology, Postgraduate Institute of Medical Education and research, Chandigarh, India, Tel +9|-|72-2755 I |4, Fax+9I-172-274440।, Email nalini203@gmail.com

Received: November II, 2016 | Published: December 29, 2016
Abbreviations: HCC, hepato cellular carcinoma; US-FNA, ultrasound-guided fine needle aspiration; IHC, immunochemistry; FL-HCC, fibro-lamellar hepato cellular carcinoma; FNAC, fine needle Aspiration cytology; AFP, alpha feto-protein; Hbsag, hepatitis b surface antigen; HCV, hepatitis c virus; MRI, magnetic resonance imaging; IVC, inferior vena cava; MGG, may grünwald- giemsa; H \& $\mathrm{E}$, hematoxylin \& eosin; CK7, cytokeratin 7

\section{Introduction}

Fibro-lamellar hepato cellular carcinoma (FL-HCC) is a variant of hepato cellular carcinoma (HCC), which accounts for less than $1 \%$ of all primary liver cancer. ${ }^{1}$ It is distinguished from conventional HCC by its distinctive clinicopathological features. Edmondson et al described it as "eosinophilic hepato cellular carcinoma with lamellar fibrosis" in $1956 .{ }^{2} \mathrm{FL}-\mathrm{HCC}$ usually occurs at a younger age than conventional HCC. ${ }^{1-3}$ Conventional HCC is most commonly associated with cirrhosis and viral hepatitis, in contrast, most of the patients of FL-HCC do not have a history of an underlying hepatic disease. ${ }^{1-3}$ FL-HCC is usually do not present initially with metastases and has a favorable prognosis than conventional HCC. ${ }^{2}$

Cyto morphological features of FL-HCC on fine needle aspiration cytology (FNAC) have been described earlier. ${ }^{4-5}$ Detection of this uncommon variant of HCC on exfoliative cytology poses diagnostic challenges to cyto pathologists, especially when there is no clinical suspicion or clinical details are not known at the time of reporting. We present a unique case of metastatic FL-HCC detected on ascitic fluid cytology and ultrasound-guided fine needle aspiration (US-FNA) of retroperitoneal lymph node. We report a rare case of FL-HCC detected on exfoliative cytology.

\section{Case presentation}

A 32-year-old male presented with loss of appetite and weight with low grade fever since 11 months, pain abdominal since 4 months and abdominal distension since one month. Patient also had hepato splenomegaly and inguinal lymphadenopathy on ultrasonography. Serum alpha feto-protein (AFP) level and HBs Ag antigen levels and anti-HCV were normal. The results of liver function tests were not available at the time of ascitic fluid cytology. Ascites fluid was sent for cytological examination to the Cyto pathology laboratory. The sample was processed by SurePath ${ }^{\mathrm{TM}}$ liquid-based cytology (LBC) technique. The smears showed predominantly a few scattered large malignant cells. These cells were polygonal in shape and showed moderate nuclear pleomorphism, round to oval large nuclei, prominent multiple nucleoli, abundant eosinophilic granular cytoplasm (Figure 1). Ascites fluid was reported as positive for malignant cells with suggestion of HCC. CT scan showed an enhancing mass in left lobe of liver with IVC showing hypo dense contents suggestive of thrombus (Figure 2). Ultrasound showed multiple retroperitoneal lymph nodes varying in size from 1 to $2.5 \mathrm{~cm}$ in diameter. Liver lesion was not very well-demarcated on ultrasonography. USG guided FNA from retroperitoneal lymph node was performed. Air-dried, MGGstained (May Grünwald- Giemsa) smears and alcohol-fixed H \& E (Hematoxylin \& Eosin) stained smears were prepared. FNAC smears were highly cellular smear and showed many individual cells and occasional aggregates of tumor tissue. Individual cells were large with low nuclear to cytoplasmic ratio, had abundant granular and eosinophilic cytoplasm with focal cytoplasmic vacuolations. Tumor cells had round nuclei, coarse chromatin, prominent nucleoli and tumor cells were seen associated with many fibro-collagenous tissue fragments (Figure 3A \& 3D). Immunochemistry was performed on cell block, which was prepared from FNA aspirate. The tumor cells were strongly positive for Hep Par 1 and cytokeratin 7 (CK7) (Figure $3 \mathrm{E} \& 3 \mathrm{~F}$ ). Considering the age of the patient [32 years], normal AFP levels, MRI findings, presence of malignant cells with hepatocytic appearance in ascitic fluid cytology and FNAC findings with IHC from retroperitoneal lymph node, a final diagnosis of metastatic FL-HCC was rendered on the USG -FNA sample and ascitic fluid cytology. 


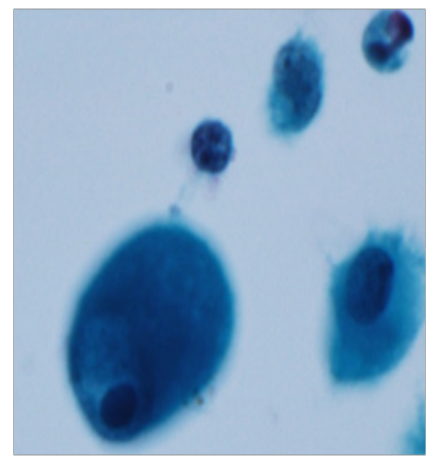

Figure I Sure Path ${ }^{T M}$ Liquid-based cytology sample of ascitic fluid showing an occasional large tumor cell with round nucleus, very prominent nucleolus and abundant granular cytoplasm and well-defined cytoplasmic borders (Papx|00X).

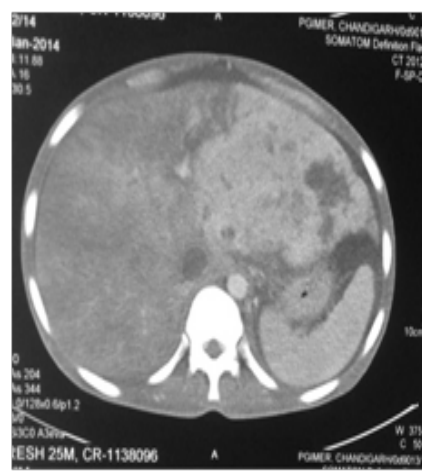

Figure $2 \mathrm{Axial} C \mathrm{CT}$ scan showing an enhancing mass in left lobe of liver with an expansible thrombus in cavoatrial junction/superior part of intra hepatic IVC.

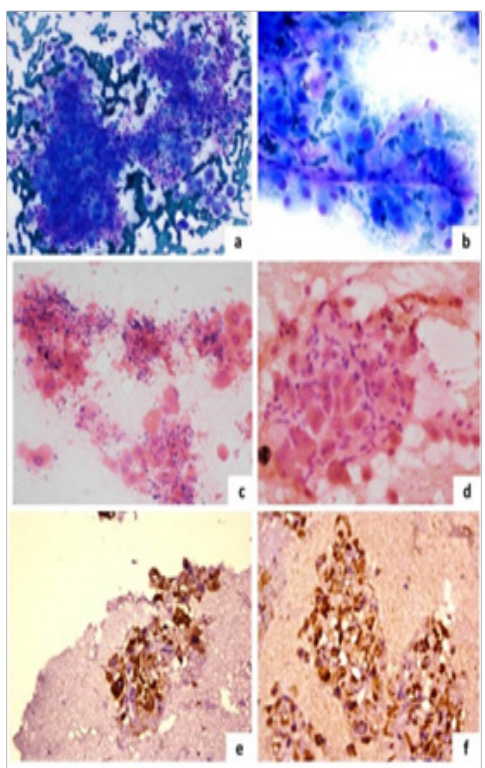

Figure 3

3A) Panel of microphotographs from FNAC from retroperitoneal lymph node; 3a- smear showing large tissue fragments of tumor cells (MGGX20X).

3B) Large hepatocytic polygonal tumor cells with capillary channels (MGGx40X).

3C) Tissue fragments comprising of large hepatocytic cells associated with fibro connective tissue (H\&Ex20X).

3D) Tumor cells showing abundant eosinophilic cytoplasm (H\&Ex40X).

3E) Tumor cells strongly positive for Hep Par I(IHCx40X); 3f- tumor cells positive for cytokeratin7(IHCx40X).

\section{Discussion}

FL-HCC is a rare morphological variant of conventional HCC which is mostly not associated with cirrhosis or other chronic liver diseases, and it has a favourable prognosis. ${ }^{2} \mathrm{HCC}$ usually is diagnosed on radiology and /or on hepatic FNAC or core biopsy. Rarely, it may present with metastases. We report a rare case of FL-HCC detected on ascitic fluid cytology prepared by SurePath ${ }^{\mathrm{TM}}$ LBC. LBC leads to cellular concentration and therefore, false negative rates are reduced remarkably. Few previous studies have described cyto morphological features of FL-HCC similar to the present case. FNAC smears in case of FL-HCC are usually variably cellular and show predominantly dispersed population of large- sized tumor cells. These cells usually have abundant eosinophilic to granular cytoplasm, centrally placed nuclei, vesicular chromatin pattern, very conspicuous nucleoli and low nuclear to cytoplasmic ratios. Cytoplasmic "pale bodies" represent non-specific cytoplasmic invaginations. ${ }^{6}$ Intra cytoplasmic hyaline globules and bile can also be present. ${ }^{5}$ Bland, spindle shaped cells arranged in parallel bands may also be noted on cytology smears, representing collagen fibers and fibroblasts, which are better appreciated on histologic sections. The trabecular arrangement with transversing capillary channels of conventional HCC is not usually seen. ${ }^{4-6}$ Some times, FL-HCC may be difficult to differentiate from conventional HCC. As compared to conventional HCC, the smears in the present case did not reveal the "basketing" pattern" or traversing capillaries that are commonly seen in conventional HCC. Because of characteristic cyto- histological features of FL-HCC's, this tumor can be readily diagnosed even in the metastatic site. Metastatic FL-HCC has previously been reported in cervical and mediastinal lymph nodes. ${ }^{7,8}$ There are a few reports in the English literature where a diagnosis of FL-HCC is rendered at uncommon sites. Kunz et al. ${ }^{9}$ reported a case with tumor involving intra hepatic bile ducts and on biliary brushings a diagnosis was suspected, however, FNAC demonstrated the features of FL-HCC. ${ }^{9}$ Use of FNAC has rarely been reported in the diagnosis of metastatic FL-HCC. ${ }^{7,8}$ Detection of F-HCC in effusion sample has been only described rarely. ${ }^{10}$ In the present case, we prepared cell block and performed IHC which was helpful to clinch the diagnosis. On IHC, the tumor cells of FL-HCC showed positivity for Hep Par 1 similar to conventional HCC but CK7 also showed strong positivity. IHC for Hep Par 1 and CK7 helped to determine the hepatic origin of the tumor, however the same cannot differentiate various variants of HCC. We report detection of fibro lamellar type of hepato cellular carcinoma in ascitic fluid cytology and the diagnosis was confirmed by US-FNA from retroperitoneal lymph node along with IHC on cell block. This case highlights role of cyto pathologist in diagnosing such uncommon tumors at unusual sites.

\section{Acknowledgements}

None.

\section{Conflict of interest}

The author declares no conflict of interest.

\section{References}

1. El Serag HB, Davila JA. Is fibro lamellar carcinoma different from hepato cellular carcinoma? A US population- based study. Hepatology. 2004;39(3):798-803.

2. Craig JR, Peters RL, Edmondson HA, et al. Fibro lamellar carcinoma of the liver: A tumor of adolescents and young adults with distinctive clinico-pathologic features. Cancer.1980;46(2):372-379 
3. Mc Larney JK, Rucker PT, Bender GN, et al. Fibro lamellar carcinoma of the liver: Radiologic-pathologic correlation. Radiographics. $1999 ; 19(2): 453-471$.

4. Perez Guillermo M, Masgrau NA, Garcia Solano J, et al. Cytologic aspect of fibro lamellar hepato cellular carcinoma in fine needle aspirates. Diagn Cytopathol. 1999;21(3):180-187.

5. Mansouri D, Van Nhieu JT, Couanet D, et al. Fibro lamellar hepato cellular carcinoma: A case report with cytological features in a sixteen-year-old girl. Diagn Cytopathol. 2006;34(8):568-571.

6. Jain M, Niveditha SR, Bharadwaj M, et al. Cytological features of fibro lamellar variant of hepato cellular carcinoma with review of literature. Cytopathology. 2002;13:179-182.
7. Nayak R, Jain SA. Rare case of fibro lamellar hepato cellular carcinoma with unusual presentation in a young indian female- a case report. International Journal of Medical Research and Review. 2014;2(4):385-387.

8. Crowe A, Knight KS, Jhala, et al. Diagnosis of metastatic fibro lamellar hepato cellular carcinoma by endoscopic ultrasound-guided fine needle aspiration. Cytojournal. 2011;8:2.

9. Kunz G Jr, Chung J, Ali SZ. Hepato cellular carcinoma - fibro lamellar variant: Cyto pathology of an unusual case. Diagn Cytopathol. 2002;26:257-261.

10. Bernieh A, Adams K, Susan Liu X, et al. Fibro lamellar hepato cellular carcinoma in ascitic fluid: A case report with cyto histological correlation. Diagn Cytopathol. 2016;44(9):757-760. 